\title{
The Effect of CYP2C9 Genotype Variants in Type 2 Diabetes on the Pharmacological Effectiveness of Sulfonylureas, Diabetic Retinopathy, and Nephropathy
}

This article was published in the following Dove Press journal:

Vascular Health and Risk Management

\author{
Meisam Saberi' \\ Zahra Ramazani ${ }^{1}$ \\ Homeira Rashidi $^{2}$ \\ Alihossein Saberi $^{3}$ \\ 'Department of Medical Chemistry, \\ School of Pharmacy, Ahvaz Jundishpur \\ University of Medical Sciences, Ahvaz, \\ Iran; ${ }^{2}$ Diabetic Research Center, Ahvaz \\ Jundishpur University of Medical \\ Sciences, Ahvaz, Iran; ${ }^{3}$ Department of \\ Medical Genetics, School of Medicine, \\ Ahvaz Jundishapur University of Medical \\ Sciences, Ahvaz, Iran
}

\begin{abstract}
Aim: Type 2 diabetes (T2D), as a major cause of morbidity and mortality, is predicted to have a prevalence of 629 million by 2045. As diabetic patients show considerable interindividual variation in response to antidiabetic treatment, this study aimed to investigate the gene polymorphism of cytochrome P450 as well as the effectiveness and safety of glibenclamide and gliclazide for different genotypes of CYP2C9. Besides, the chronic side effects of T2D including retinal microvasculature complications or retinopathy and renal dysfunction due to nephropathy in different genotypes were considered.
\end{abstract}

Patients and Methods: The participants including 80 T2D patients treated with glibenclamide or gliclazide were recruited from university hospitals of Ahvaz Jundishpur University of Medical Sciences, Ahvaz, in the southwest of Iran. Blood samples were collected from the patients at $2.5 \mathrm{~h}$ after the morning dose of glibenclamide and $12 \mathrm{~h}$ after the last dose of gliclazide. Genotyping from the extracted DNA was, then, performed using PCR-RFLP. The plasma level of glibenclamide and gliclazide was, in turn, measured by the reverse-phase high-pressure liquid chromatography.

Results: The results showed that the wild-type allele, i.e., CYP2C $* 1$, occurred in the highest frequency $(0.8)$, while the frequency rates of the mutant allele, i.e., CYP2C $9 * 2$ and $\mathrm{CYP} 2 \mathrm{C} 9 * 3$, were 0.15 and 0.05 , respectively. Moreover, no significant association was found between any of the genotypes as well as the clinical and biochemical characteristics of the patients. The findings also showed that the plasma level of sulfonylureas (i.e., glibenclamide and gliclazide) was the highest in the patients with the CYP2C9*3 allele. It was also found that $75.9 \%$ of the patients with variant genotypes had experienced hypoglycemia events. Furthermore, in the absence of wild type allele, a significant increase was observed in retinopathy $(\mathrm{p}=0.039)$ and nephropathy $(\mathrm{p}=0.05)$.

Conclusion: The findings can provide guidelines for the optimal management of the treatment protocols with sulfonylurea intended to control the T2D complications.

Keywords: CYP2C9, glibenclamide, gliclazide, retinopathy, nephropathy

\section{Introduction}

Type 2 diabetes (T2D), as a metabolic disorder characterized by hyperglycemia, is reported to have a prevalence of 629 million by $2045 .{ }^{1}$ Genetic factors are, in turn, supposed to contribute to great inter-individual variation in response to antidiabetic treatment. Indeed, the importance of pharmacogenetics to reinforce therapeutic outcomes is widely acknowledged. Among others, sulfonylureas, as a group of
Department of Medical Genetics, School of Medicine, Ahvaz Jundishapur University of Medical Sciences, Ahvaz, Iran

Tel +98-916II33488

Fax +98-6133332036

Email saberi-a@ajums.ac.ir 
antidiabetic drugs, are most widely used for the treatments of patients with T2D. ${ }^{2}$ In effect, the sulfonylurea receptor functions as a part of the ATP-dependent potassium channel in the beta cells of the pancreas (K-ATP). ${ }^{3}$ This way, channel function is inhibited due to the binding of the receptor to sulfonylurea. This, in turn, alters the resting potential of the cell, resulting in calcium influx and the stimulation of insulin secretion.

The net effect occurs when the beta cell response increases in both stimulants of glucose and non-glucose (e.g., amino acids), and insulin secrets at higher levels at all blood glucose concentrations. Having similar characteristics in their structure, glibenclamide and gliclazide, as the second generation sulfonylureas, are prescribed in much lower doses than the previous generation of sulfonylureas. However, the effectiveness of different sulfonylureas in lowering blood glucose concentration is reported to be the same. ${ }^{2}$ Although sulfonylureas are usually welltolerated, they usually have serious side effects including hypoglycemia which is more common in the long-acting version of such drugs. ${ }^{4-6}$

Sulfonylurea is known to be metabolized by cytochrome P450, which is encoded by the CYP2C9 gene. Several studies have already reported medical evidence suggesting that in patients with CYP2C9*2 and CYP2C $9 * 3$ variant alleles, the metabolism of the substrate is significantly slower than that in the wild-type allele. ${ }^{7-10}$ For instance, in a Go-DART study by Zhou et $\mathrm{al}^{11}$, it was shown that sulfonylureas were metabolized mainly by the cytochrome P450 2C9 (15). Two CYP2C9* and CYP2C *3 genotypes were, indeed, found to be associated with impaired substrate metabolism. Furthermore, many reports have demonstrated that at least one variant allele causes a significant decrease in CYP2C9 activity, requiring dose adjustment for sulfonylureas. ${ }^{12-15}$ Therefore, CYP2C9 gene polymorphism is likely to affect the metabolism and the adverse drug reaction (ADR) of glibenclamide and gliclazide.

Diabetic retinopathy, a retinal vascular disorder, occurs as a complication of diabetes mellitus and causes blindness in diabetic patients. In effect, the associated vision loss results from several mechanisms, including neovascularization, macular edema, and retinal capillary nonperfusion. ${ }^{16}$ Likewise, a few studies have shown that diabetic retinopathy is influenced by CYP2C9 variants. ${ }^{17}$ Epoxyeicosatrienoic acids (EETs) produced by CYP2C8 and CYP2C9, which are hydrolyzed to dihydroxyeicosatrienoic acids (DHETs) by epoxide hydrolase, are known to play an important role in the diabetic retinopathy. ${ }^{18}$ Therefore, CYP2C9 genetic polymorphism is also likely to affect retinopathy, considering that renal dysfunction has been reported in patients with T2D. The severity and onset of nephropathy maybe thus influenced by genetic variants.

This study was, thus, intended to analyze the frequency of CYP2C9 genotypes in $\mathrm{T} 2$ diabetic patients, and also investigate the metabolism as well as safety (ADR) of glibenclamide and gliclazide in patients with different CYP2C9 genotypes to predict their response to sulfonylureas. Also, this study examined whether the CYP2C9 polymorphisms were associated with the risk of diabetic retinopathy and diabetic nephropathy.

\section{Patients and Methods Patient Selection and Clinical Study Design}

Iranian patients with T2D who had received medical treatment with sulfonylureas (i.e., glibenclamide or gliclazide) at Golestan and Imam Khomeini Hospitals were recruited for this study. The participants included 80 T2D male and female patients who had to take $10 \mathrm{mg}$ Glibenclamide and $160 \mathrm{mg}$ Gliclazide for more than 3 months. Blood samples were collected from the patients at $2.5 \mathrm{~h}$ after the morning dose of glibenclamide ${ }^{15}$ and $12 \mathrm{~h}$ after the last dose of gliclazide, corresponding to the time for the peak drug concentration. ${ }^{19}$

The plasma samples collected were used to estimate the levels of sulfonylureas, and the buffy coat fraction was used for DNA extraction and genotyping of variant alleles. The plasma levels of glibenclamide and gliclazide were, then, estimated by reverse-phase high-pressure liquid chromatography. ${ }^{20,21}$ The DNA was extracted from the buffy coat using the salting-out extraction procedure. The variant alleles CYP2C9*2 and *3 were genotyped by PCR- restriction fragment length polymorphism (RLFP). ${ }^{22}$ Fasting blood glucose (FBS), HbAlc, blood urea nitrogen (BUN), creatinine (Cr), microalbuminuria, LDL (Low-density lipoprotein), HDL (High-density lipoprotein), total cholesterol and retinopathy data were collected from the patients' medical records. Nephropathy was, in turn, achieved by using an NKF KDOQI.

GUIDELINES. ${ }^{23}$ The cause of chronic kidney disease, glomerular filtration rate (GFR) and albuminuria, collectively referred to as CGA staging, was checked according to the guidelines. The GFR $\geq 90 \mathrm{~mL} / \mathrm{min} / 1.73 \mathrm{~m}^{2}$ (G1) was considered as normal or high GFR, while GFR $\leq 15$ (G5) was described as kidney failure. There are several categories between G1 and G5 (i.e., G2, G3a, G3b, and G4) meaning mildly to severely decreased as GFR. Increasing albuminuria 
from less than $30 \mathrm{mg} / \mathrm{g}$ to more than $300 \mathrm{mg} / \mathrm{g}$ was, in turn, categorized as A1 to A3 (normal to severely increased). We described the risk of nephropathy using the combination of GFR decreasing and albuminuria increasing ranges. For instance, the patients with GFR $<15 \mathrm{~mL} / \mathrm{min} / 1.73 \mathrm{~m}^{2}$ and albuminuria more than $300 \mathrm{mg} / \mathrm{g}$ (G5-A3) were considered at very high risk for nephropathy.

This study was approved by the Medical Ethics Committee at Ahvaz Jundishapur University of Medical Sciences, Iran. Informed written consent was obtained from the patients after they were introduced to the research objectives and the procedure used.

\section{Statistical Analysis}

In the statistical analysis performed, all quantitative data were presented as mean \pm standard deviation (SD), and the comparison between different genotype groups was conducted using an independent $t$-test and ANOVA test. The chi-square test was, in turn, used to compare the genotype and allelic frequency distribution of CYP2C9, as well as the distribution of sulfonylureas plasma level, ADRs (hypoglycemia), and the retinopathy and nephropathy between the genotype groups.

\section{Results}

A total of 80 patients with T2D were genotyped by PCRRFLP method described previously (Table 1). The compound heterozygous genotypes were scored according to the Seng et al paper. ${ }^{22}$ In CYP2C9*1/CYP2C9*2 genotype, Avall digestion produced $296 b+79 b p$ and $375 b p$, while Kpn1 digestion created 105bp. In turn, in CYP2C9*1/CYP2C9*3 genotype, AvaII digestion produced 375bp and 296bp $+79 \mathrm{bp}$, while $K p n 1$ digestion produced $85 \mathrm{bp}+20 \mathrm{bp}$. Likewise, in the CYP2C9*2/CYP2C9*3 genotype, AvalI digestion produced $375 b p$ and $296 b p+79$ bp, while Kpnl digestion produced $105 \mathrm{bp}$ fragments.

The genotype and allelic frequencies obtained were consistent with those of Hardy-Weinberg Equilibrium. Indeed, CYP2C9*1 (wild type) was the predominant allele and occurred in high frequency in this study (0.8). Besides, the frequency of mutant alleles, CYP2C9*2 and CYP2C9*3, were 0.15 and 0.05 , respectively.

The results also showed that there were 51 patients with CYP2C9*1/*1 genotype (63.57\%), and CYP2C9*1/*2 $(22.5 \%)$ was found to be the second most frequent genotype. Also, the percentage rates of the observed CYP2C $9 * 1 / * 3$ and CYP2C9*2/*3 genotypes were 10 and 2.5 , respectively. Moreover, only one patient was observed with the CYP2C9*2/*3 compound heterozygous genotype. Though none of the patients was a homozygous mutant genotype for the CYP2C9*3 allele, two patients with CYP2C9*2 allele were found to be a homozygous mutant.

The demographic information and biochemical characteristics of the patients are summarized in Table 1. The parameters such as age, gender, duration of diabetes, FBS, HbA1c, BUN, TG, LDL, HDL, and total cholesterol were compared in different genotypes. The findings showed that there was no significant correlation between any of the genotypes as well as the clinical and biochemical characteristics of the patients. It was also found that 55 patients, out of 80 patients, underwent concomitant treatment with two drugs, namely sulfonylureas (glibenclamide or gliclazide) and metformin, 16 patients with three drugs (i.e., metformin, sulfonylureas, and another antidiabetic drug),

Table I The Demographic and Biochemical Factors in Different Genotypes of CYP2C9

\begin{tabular}{|c|c|c|c|c|c|c|}
\hline & $\begin{array}{l}\text { CYP2C } 9 * I / * I \\
(n=5 I)\end{array}$ & $\begin{array}{l}\text { CYP2C } 9 * 1 / * 2 \\
(n=18)\end{array}$ & $\begin{array}{l}\text { CYP2C9*I/*3 } \\
(n=8)\end{array}$ & $\begin{array}{l}\text { CYP2C } 2 * 2 / * 2 \\
(n=2)\end{array}$ & $\begin{array}{l}\text { CYP2C } 9 * 2 / * 3 \\
(n=1)\end{array}$ & p-value \\
\hline Age (years) & 58 & 54 & 56 & 51 & 53 & $\mathrm{~N} / \mathrm{A}$ \\
\hline Gender (M/F) & $18 / 33$ & $9 / 9$ & $\mathrm{I} / 7$ & $0 / 2$ & $0 / 1$ & $\mathrm{~N} / \mathrm{A}$ \\
\hline Duration of diabetes (years) & 10 & 8 & 11 & 11 & 6 & 0.318 \\
\hline $\mathrm{FBS}(\mathrm{mg} / \mathrm{dl})$ & 185 & 202 & 196 & 109 & 162 & 0.666 \\
\hline HbAlc (\%) & 8.3 & 8 & 8.4 & 6.6 & 8.5 & 0.711 \\
\hline BUN & 14 & 15 & 18 & 12 & 9 & 0.387 \\
\hline Creatinine & 0.92 & 0.95 & I.I & 0.8 & 0.8 & 0.238 \\
\hline TG & 156 & 176 & 260 & 260 & 115 & 0.316 \\
\hline LDL & 96 & 100 & 132 & 125 & 63 & 0.157 \\
\hline HDL & 44 & 51 & 39 & 40 & 33 & 0.177 \\
\hline Total cholesterol & 167 & 173 & 193 & 181 & 119 & 0.498 \\
\hline
\end{tabular}


9 patients with monotherapy, 5 patients with gliclazide, and other 4 patients with glibenclamide.

To examine the effects of CYP2C9 genotypes on the plasma level of sulfonylureas, the drug concentration was investigated using HPLC 2.5 hours after glibenclamide and 12 hours after gliclazide administration. The results showed that a relatively higher plasma level of sulfonylureas was observed in the CYP2C9*3 allele in comparison to those of other alleles. Besides, it was found that there was no steadystate in the plasma level of the drugs in patients with wild-type alleles (Table 2). Furthermore, the distributions of various alleles in two groups of patients, namely, steady-state and nonsteady-state, were significantly different $(p=0.001)$.

Additionally, the occurrence of hypoglycemic ADRs to sulfonylureas was compared between the wild-type and variant genotypes. The results showed that $75.9 \%$ of the patients with variant genotypes had experienced hypoglycemia status, whereas this was not observed in the wild-type genotypes. Overall, these data showed that hypoglycemia status increased significantly in the patients with variant genotypes. The retinopathy was, in turn, checked in all samples, and a significant association was identified between the duration of diabetes and retinopathy $(\mathrm{p}=0.002)$.

To investigate the association between the variant genotypes and retinopathy, we initially checked the average duration of diabetes between various genotypes. As the findings showed, no significant difference was observed $(\mathrm{p}=0.318)$. However, the association between the different genotypes and retinopathy was found to be significant $(\mathrm{p}=0.039)$. In the patients with CYP2C $9 * 1 / * 3$ genotype $50 \%$, while in CYP2C $9 * 2 / * 2$ and $* 2 / * 3$ genotypes, $100 \%$ of the patients were affected by retinopathy (Figure 1).

In turn, to evaluate the impact of the duration of diabetes on the renal function, the correlation between nephropathy and the duration of diabetes was investigated. As the results showed, no significant correlation was found $(p=0.404)$. However, patients with variant genotypes were found to be significantly affected by nephropathy $(p=0.05)$. Besides,
$50 \%$ of the patients with CYP $2 \mathrm{C} 9 * 1 / * 3$ and $67 \%$ of the patients with $\mathrm{CYP} 2 \mathrm{C} 9 * 2 / * 2$ and $\mathrm{CYP} 2 \mathrm{C} 9 * 2 / * 3$ genotypes were identified to be affected (Figure 2).

\section{Discussion}

This study was, initially, intended to investigate the frequency of variant genotypes in 80 patients with T2D. Then, the effect of variant genotypes of CYP2C9 on responses to sulfonylureas including glibenclamide and gliclazide was examined. The association between hypoglycemic ADRs and variant genotypes in $\mathrm{T} 2$ diabetics who were treated with sulfonylureas (glibenclamide or gliclazide) was also sought. Finally, the influence of variant genotypes on diabetic nephropathy and retinopathy was studied.

Investigating the prevalence of different CYP2C9 genotypes, it was found that the wild-type allele (CYP2C9*1) was the highest. This result is similar to the allelic frequency reported in the study conducted by Ataby et al in the north of Iran, involving patients from Turkman and Fars ethnic groups. ${ }^{24}$

Similarly, several other studies showed that the most prevalent allele of CYP2C9*1 was the wild-type allele. ${ }^{9,25-}$ 36 Also, it was reported that the second most prevalent allele was the CYP2C9*2 allele (15\%), which is consistent with the previous reports in Caucasian populations. ${ }^{37}$ However, the lowest frequency of this allele was observed in the Africans (0.46\%), East Asians (0.56\%) and Native Americans (1.25\%). ${ }^{37}$ CYP2C9*3 allele in our study was found to be $5 \%$, similar to the allelic frequency observed in Ataby for Turkman and Fars ethnic groups. ${ }^{24}$ In contrast, CYP2C9*3 showed the highest frequency among the South Asians (11.7\%). ${ }^{37}$ Moreover, the frequency of the CYP2C9*3/*3 homozygous genotype was not identified in our study as well as in the Caucasian ethnic groups, ${ }^{38,39}$ whereas it was detected frequently in the Malay and Indian ethnic groups. $^{22}$ Overall, these data show that the allelic frequency of the Iranian ethnic groups is similar to that of the Caucasian population.

Table 2 Plasma Level of Glybenclamid and Glyclaside as Well as the Number of Patients Reached Steady State in Different Genotype of CYP2C9

\begin{tabular}{|l|l|l|l|l|l|}
\hline & CYP2C9*I/*I & CYP2C9*I/2 & CYP2C9*I/*3 & CYP2C9*2/*2 & CYP2C9*2/*3 \\
\hline Plasma level of glibenclamide $(\mu \mathrm{g} / \mathrm{mL})$ & 0.0053 & 0.1695 & 0.8072 & 0.6990 & N/A \\
Plasma level of gliclazide $(\mu \mathrm{g} / \mathrm{mL})$ & 0.0427 & 0.2213 & 2.4696 & 0.4790 & 2.4620 \\
Steady state (\# cases) & 0 & 4 & 7 & 1 & $1 *$ \\
Non-steady state (\# cases) & $5 \mathrm{I}$ & 14 & $\mathrm{I}$ & $\mathrm{I}$ \\
\hline
\end{tabular}

Note: $*$ The distributions of various alleles in two groups of patient, i.e., steady-state and non-steady-state, were significantly different $(p=0.00 \mathrm{I})$. 


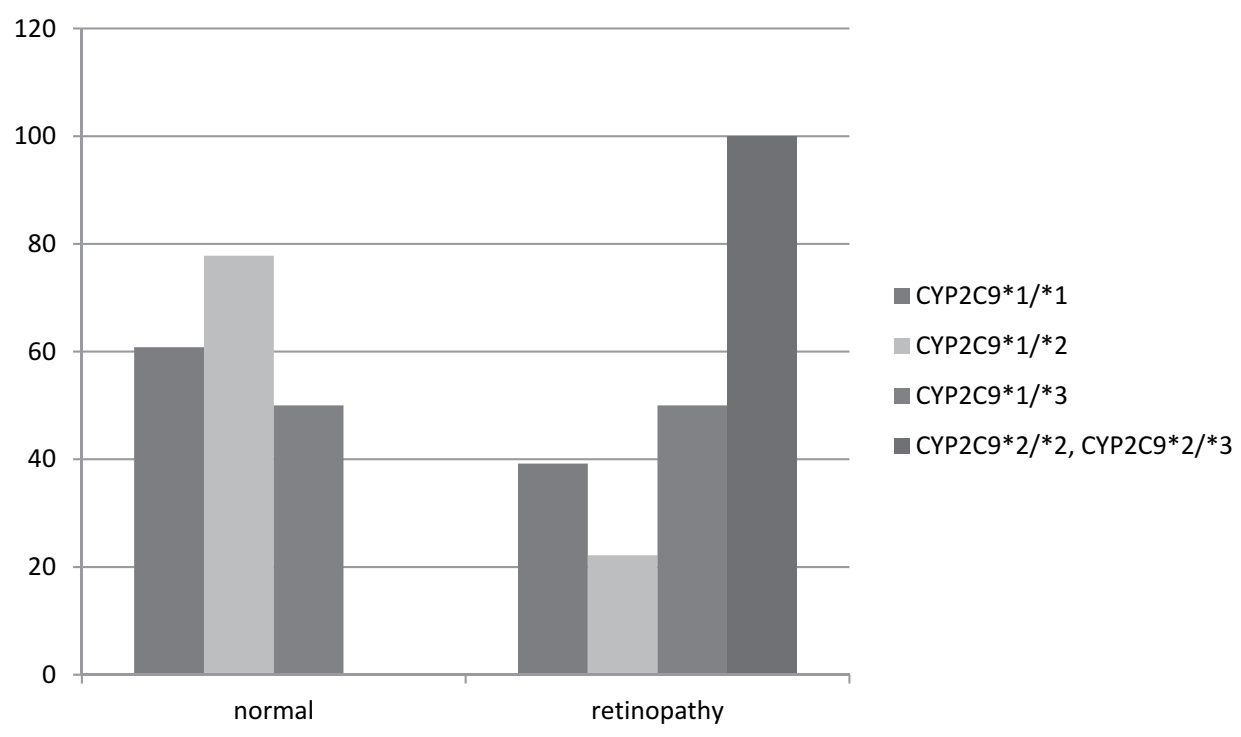

Figure I Association of retinopathy and different genotypes of CYP2C9.

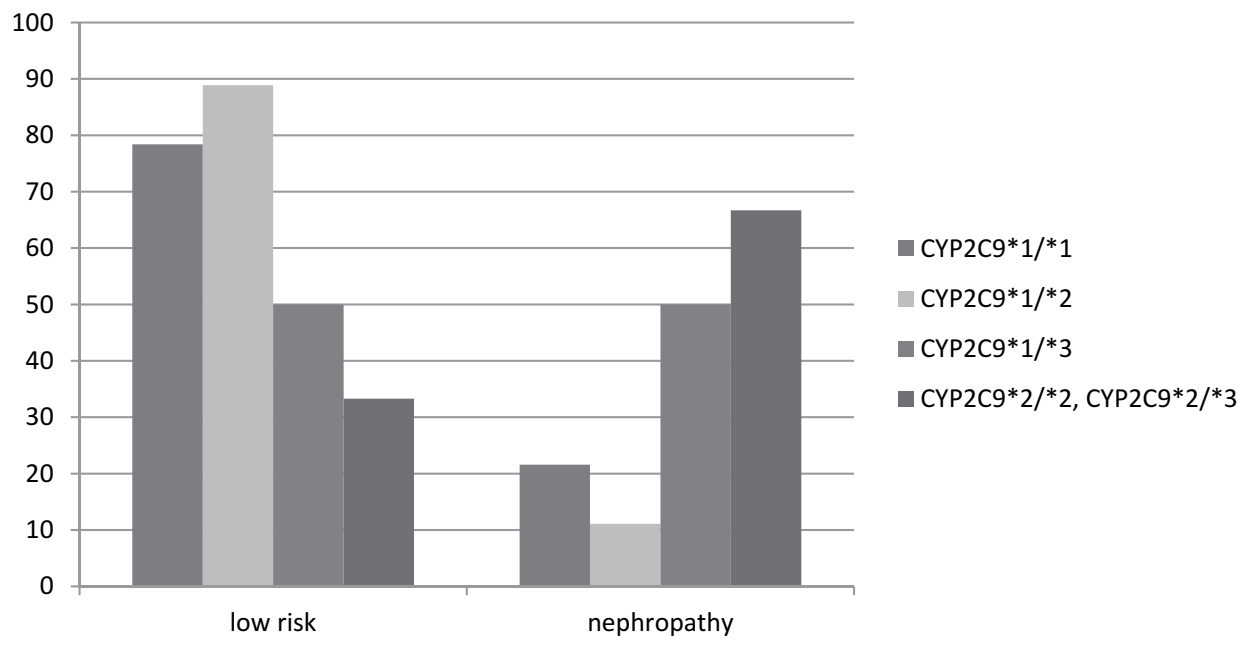

Figure 2 Association of nephropathy and different genotypes of CYP2C9.

To find out the different responses of glibenclamide treatment in the patients with various CYP2C9 genotypes, the plasma level of glibenclamide in $\mathrm{T} 2$ diabetic patients with $\mathrm{CYP} 2 \mathrm{C} 9 * 1 / * 1, \quad$ CYP2C $9 * 1 / 22, \quad$ CYP $2 \mathrm{C} 9 * 1 / * 3$, $\mathrm{CYP} 2 \mathrm{C} 9 * 2 / * 2$, and $\mathrm{CYP} 2 \mathrm{C} 9 * 2 / * 3$ genotypes was measured 2.5 hours after the treatment. The plasma concentration of gliclazide was also measured for the same genotypes 12 hours after the treatment. Given that the peak of plasma concentration in the previous studies reached a maximum of 2 hours after the glibenclamide treatment, ${ }^{15}$ the plasma level was measured 2.5 hours after the administration in our study. However, as gliclazide remains in plasma for a longer time, the measurement was, thus, done 12 hours after the administration.
Consistent with the previous studies, the highest plasma level of glibenclamide was observed in the CYP $2 \mathrm{C} 9 * 1 / * 3$ $(0.8 \mu \mathrm{g} / \mathrm{mL})$. Moreover, the plasma level of gliclazide was higher not only in the CYP2C $9 * 1 / * 3$ genotype but also in CYP2C $9 * 2 / * 3$ genotypes $(2.46 \mu \mathrm{g} / \mathrm{mL})$. In contrast, the lowest level of glibenclamide and gliclazide concentration in the plasma was observed in the wild-type CYP2C $9 * 1 /$ *1 genotype. Therefore, this result implies that the elimination of plasma glibenclamide and gliclazide in patients with $\mathrm{CYP} 2 \mathrm{C} 9 * 1 / * 3$ may be slower than that in patients with CYP2C $9 * 1 / * 1$. This, in turn, indicates that the higher concentration of glibenclamide and gliclazide may cause stronger pharmacological effects. Moreover, it was found that the plasma concentration of glibenclamide and 
gliclazide in other variant genotypes, namely CYP2C9*1/ $* 2$ and $\mathrm{CYP} 2 \mathrm{C} 9 * 2 / * 2$ was higher than that in the wildtype genotype (Table 2). However, the plasma level of gliclazide in CYP2C9*2/*2 was similar to that in the wildtype CYP2C $9 * 1 / * 1$ genotypes. Overall, these data showed that in the patients with wild-type genotypes, the elimination of glibenclamide and gliclazide was likely to be faster than that invariant genotypes, as a lower concentration of such drugs is needed for the patients with wild-type genotypes.

The association of variant genotypes with hypoglycemic ADRs in T2 diabetic patients treated with the sulfonylureas glibenclamide or gliclazide was, in turn, investigated. The results showed that the presence of variant genotypes predisposes sulfonylurea-treated patients to hypoglycemia. It was found that in individuals with variant genotypes (CYP2C9*1/*2, CYP2C9*1/*3, CYP2C9*2/*2, CYP2C9*2/*3) when treated with sulfonylureas, the risk of suffering a hypoglycemic event significantly increased. Similarly, in a study with a limited number of patients (i.e., 20 patients), Holstein et al reported that $\mathrm{CYP} 2 \mathrm{C} 9 * 2 / * 3$ and $\mathrm{CYP} 2 \mathrm{C} 9 * 1 / * 3$ genotypes correlated with severe hypoglycemia. ${ }^{40}$ By contrast, in the study conducted by Surendiran et al, there was no significant difference in the occurrence or severity of hypoglycemia. $^{41}$ Accordingly, since hypoglycemia can significantly cause morbidity and mortality, the treatment protocol for diabetes should be geared to the patients' individual needs to reduce the occurrence of hypoglycemic episodes. It is suggested that, particularly at the beginning of the treatment by sulfonylurea, genotype analysis is likely to prevent overdose in patients with CYP2C9 genotypes, causing lower activity. ${ }^{42}$ In effect, identifying the genotype of the patient might help the physician adjust the regimen protocol accordingly, and therefore reduce the possibility of the patient's experiencing a hypoglycemic event. This approach to CYP2C9 genotyping of patients before the treatment by glimepiride has already been adopted in Korea and Russia. ${ }^{43,44}$ Our data also provide evidence supporting the use of such an approach.

\section{Limitations}

There are several limitations to consider in this study, including the small sample size, compared to Go-DART trials $^{11}$ studies, limiting the generalizability of the findings. Indeed, despite registering180 patients for the research purpose, we could only include data from 82 patients in the final analysis as some of the patients did not cooperate to be compatible with our inclusion criteria. However, the association of plasma level of sulfonylurea with CYP2C9 genotypes was statistically significant ( $p$-value less than 0.05). Moreover, future studies using large mega studies seem necessary to confirm our findings.

To the best of our knowledge, this may be the first study on the impact of CYP2C9 genotype variants on retinopathy in patients with T2D. The data showed that in the absence of wild-type alleles, all patients were affected by retinopathy, while the presence of wild-type allele delayed the microvascular complications (Figure 1). However, it is not clear how CYP2C9 variant alleles (*2 and *3) influenced the retinal microvasculatures, but a few studies have reported the influence of CYP2C9 and their products, namely the epoxyeicosatrienoic acids (EETs), in the hypoxia-induced VEGF production and pathologic retinal angiogenesis. ${ }^{45}$ Also, comparing the risk of nephropathy in the patients with different CYP2C9 alleles, based on the marked increase in microalbuminuria and the decrease observed in the glomerular filtration rate (GFR), $66.7 \%$ of the patients with CYp2C9*2/*2 and CYP2C9*2/*3 genotypes were identified to be affected by nephropathy (Figure 2). It seems that the presence of wild-type alleles $(* 1 / * 1, * 1 / * 2$ and $* 1 / * 3$ ) protected the normal function of the kidney in patients with T2D. However, the precise mechanism of CYP2C9 in diabetic retinopathy and nephropathy is still unclear.

It is concluded that the genotype analysis of CYP2C9 in patients with $\mathrm{T} 2 \mathrm{D}$ can provide guidelines for the optimal management of the treatment protocol with sulfonylurea by physicians so that they can make sound decisions to regulate the treatment protocol accordingly before the initiation of sulfonylurea therapy, and thus prevent the hypoglycemic events.

\section{Ethics Approval and Consent to Participate}

In this clinical experimental study, all peripheral blood samples were collected and used by the ethical rules confirmed by the ethics committee of Ahvaz Jundishapur University of Medical Sciences (Code no: IR.AJUMS. REC.139.221). Ethical issues (including plagiarism, informed consent, misconduct, data fabrication and/or falsification, double publication and/or submission, redundancy, etc.) have been completely observed by the authors. Written informed consent was also obtained 
from all patients. Data were anonymously and confidentially retrieved with compliance with the Declaration of Helsinki.

\section{Acknowledgments}

We thank the patients and nurses in diabetic specific clinics at Imam Khomeini and Golestan Hospitals affiliated to Ahvaz Jundishpur University of Medical Sciences, Ahvaz, Iran for their contribution to the research. The study was supported by the grant provided by the Deputy of Research Affairs at Ahvaz Jundishpur University of Medical Sciences.

\section{Author Contributions}

All authors contributed to data analysis, drafting or revising the article, gave final approval of the version to be published, and agree to be accountable for all aspects of the work.

\section{Disclosure}

The authors declare no conflicts of interest.

\section{References}

1. Federation ID, editor. IDF Diabetes Atlas Update Poster. Brussels, Belgium: International Diabetes Federation; 20152015.

2. Bressler R, Johnson DG. Pharmacological regulation of blood glucose levels in non-insulin-department diabetes mellitus. Arch Intern Med. 1997;157(8):836-848. doi:10.1001/archinte.1997.00440290014001

3. Aguilar-Bryan L, Nichols CG, Wechsler SW, Clement JP IV. Cloning of the beta cell high-affinity sulfonylurea receptor: a regulator of insulin secretion. Science. 1995;268(5209):423. doi:10.1126/science.7716547

4. Tessier D, Dawson K, Tetrault J, Bravo G, Meneilly G. Glibenclamide vs gliclazide in type 2 diabetes of the elderly. Diabet Med. 1994;11(10):974-980. doi:10.1111/j.1464-5491.1994.tb00256.x

5. Holstein A, Plaschke A, Egberts EH. Lower incidence of severe hypoglycemia in patients with type 2 diabetes treated with glimepiride versus glibenclamide. Diabetes Metab Res Rev. 2001;17 (6):467-473. doi:10.1002/dmrr.235

6. Gangji AS, Cukierman T, Gerstein HC, Goldsmith $\mathrm{CH}$, Clase CM. A systematic review and meta-analysis of hypoglycemia and cardiovascular events. Diabetes Care. 2007;30(2):389-394. doi:10.2337/dc061789

7. Lee CR, Goldstein JA, Pieper JA. Cytochrome P450 2C9 polymorphisms: a comprehensive review of the in-vitro and human data Pharmacogenet Genomics. 2002;12(3):251-263. doi:10.1097/0000 8571-200204000-00010

8. Takanashi K, Tainaka H, Kobayashi K, Yasumori T, Hosakawa M, Chiba K. CYP2C9 Ile359 and Leu359 variants: enzyme kinetic study with seven substrates. Pharmacogenet Genomics. 2000;10 (2):95-104. doi:10.1097/00008571-200003000-00001

9. Sullivan-Klose TH, Ghanayem BI, Bell DA, et al. The role of the CFP2C9-Leu 359 allelic variant in the tolbutamide polymorphism. Pharmacogenet Genomics. 1996;6(4):341-349. doi:10.1097/00008 571-199608000-00007

10. Inoue K, Yamazaki H, Imiya K, Akasaka S, Guengerich FP, Shimada T. Relationship between CYP2C9 and 2C19 genotypes and tolbutamide methyl hydroxylation and S-mephenytoin 4'-hydroxylation activities in livers of Japanese and Caucasian populations. Pharmacogenet Genomics. 1997;7(2):103-113. doi:10.1097/00008571-199704000-00003
11. Zhou K, Donelly L, Burch L, et al. Loss-off-functionCYP2C9 variants improve therapeutic response to sulfonylureas in type 2 diabetes: a go-DARTS study. Clin Pharmacol Ther. 2010;87(1):52-56. doi:10.1038/clpt.2009.176

12. Kirchheiner J, Bauer S, Meineke I, et al. Impact of CYP2C9 and CYP2C19 polymorphisms on tolbutamide kinetics and the insulin and glucose response in healthy volunteers. Pharmacogenet Genomics. 2002;12(2):101-109. doi:10.1097/00008571-200203000-00004

13. Shon J-H, Yoon Y-R, Kim K-A, et al. Effects of CYP2C19 and CYP2C9 genetic polymorphisms on the disposition of and blood glucose lowering response to tolbutamide in humans. Pharmacogenet Genomics. 2002;12 (2):111-119. doi:10.1097/00008571-200203000-00005

14. Suzuki K, Yanagawa T, Shibasaki T, Kaniwa N, Hasegawa R, Tohkin M. Effect of CYP2C9 genetic polymorphisms on the efficacy and pharmacokinetics of glimepiride in subjects with type 2 diabetes. Diabetes Res Clin Pract. 2006;72(2):148-154. doi:10.1016/j.diabres.2005.09.019

15. Niemi M, Cascorbi I, Timm R, Kroemer HK, Neuvonen PJ, Kivistö KT. Glyburide and glimepiride pharmacokinetics in subjects with different CYP2C9 genotypes. Clin Pharmacol Ther. 2002;72 (3):326-332. doi:10.1067/mcp.2002.127495

16. American Academy of Ophthalmology. Preferred Practice Pattern: Diabetic Retinopathy. September 20; 2014; San Francisco, Calif: American Academy of Ophthalmology.

17. Capozzi ME, McCollum GW, Penn JS. The role of cytochrome P450 epoxygenases in retinal angiogenesis cytochrome P450 epoxygenases. Invest Ophthalmol Vis Sci. 2014;55(7):4253-4260. doi:10.1167/iovs.1414216

18. Suzuki S, Oguro A, Osada-Oka M, Funae Y, Imaoka S. Epoxyeicosatrienoic acids and/or their metabolites promote hypoxic response of cells. J Pharmacol Sci. 2008;108(1):79-88. doi:10.1254/ jphs.08122FP

19. Shao H, Ren X, Liu N, et al. Influence of CYP2C9 and CYP2C19 genetic polymorphisms on pharmacokinetics and pharmacodynamics of gliclazide in healthy Chinese Han volunteers. J Clin Pharm Ther. 2010;35(3):351-360. doi:10.1111/j.1365-2710.2009.01134.x

20. Rajendran S, Philip B, Gopinath R, Suresh B. RP-HPLC method for the estimation of glibenclamide in human serum. Indian J Pharm Sci. 2007;69(6):796. doi:10.4103/0250-474X.39436

21. Venkatesh P, Harisudhan T, Choudhury H, Mullangi R, Srinivas NR. Simultaneous estimation of six anti-diabetic drugs-glibenclamide, gliclazide, glipizide, pioglitazone, repaglinide and rosiglitazone: development of a novel HPLC method for use in the analysis of pharmaceutical formulations and its application to human plasma assay. Biomed Chromatogr. 2006;20(10):1043-1048. doi:10.1002/bmc.635

22. Seng KC, Gin GG, Sangkar JV, Phipps ME. Frequency of cytochrome P450 2C9 (CYP2C9) alleles in three ethnic groups in Malaysia. Asia Pac J Mol Biol Biotechnol. 2003;11(2):83-91.

23. Levey AS, Coresh J, Balk E, et al. National kidney foundation practice guidelines for chronic kidney disease: evaluation, classification, and stratification. Ann Intern Med. 2003;139(2):137-147. doi:10.7326/0003-4819-139-2-200307150-00013

24. Ataby OA, Tabari RG, Mansourian AR, Samai NM, Marjani A. Genetic polymorphism of cytochrome P450 2C9 (CYP2C9) in two ethnic groups in Iran. Am J Biomed Sci. 2013;5(3):177-187. doi:10.5099/aj130300177

25. Wang S-L, Huang J-D, Lai M-D, Tsai -J-J. Detection of CYP2C9 polymorphism based on the polymerase chain reaction in Chinese. Pharmacogenet Genomics. 1995;5(1):37-42. doi:10.1097/00008571199502000-00004

26. Stubbins MJ, Harries LW, Smith G, Tarbit MH, Wolf CR. Genetic analysis of the human cytochrome P450 CYP2C9 locus. Pharmacogenet Genomics. 1996;6(5):429-439. doi:10.1097/000 08571-199610000-00007

27. Nasu K, Kubot T, Ishizaki T. Genetic analysis of CYP2C9 polymorphism in a Japanese population. Pharmacogenet Genomics. 1997;7(5):405-409. doi:10.1097/00008571-199710000-00011 
28. Ackermann E, Cascorbi I, Sachse C, Brockmoller J, Mrozikiewiza P, Roots I. Frequencies and the allelic linkage of CYP2C9 mutations in a German population and the detection of a C/T mutation in intron 2. Eur J Clin Pharmacol. 1997;52:A71.

29. Kimura M, Ieiri I, Mamiya K, Urae A, Higuchi S. Genetic polymorphism of cytochrome P450s, CYP2C19, and CYP2C9 in a Japanese population. Ther Drug Monit. 1998;20(3):243-247. doi:10.1097/00007691-199806000-00001

30. Yasar Ü, Eliasson E, Dahl M-L, Johansson I, Ingelman-Sundberg M, Sjöqvist F. Validation of methods for CYP2C9 genotyping: frequencies of mutant alleles in a Swedish population. Biochem Biophys Res Commun. 1999;254(3):628-631. doi:10.1006/bbrc.1998.9992

31. Brockmöller J, Bauer S, Sachse C, et al. Frequency of cytochrome P450 CYP2C9 variants in a Turkish population and functional relevance for phenytoin. Br J Clin Pharmacol. 1999;48(3):409-415. doi:10.1046/j.1365-2125.1999.00012.x

32. Gaedigk A. Interethnic differences of drug-metabolizing enzymes. Int J Clin Pharmacol Ther. 2000;38(2):61-68. doi:10.5414/CPP38061

33. Kim J, Gaedigk A, Leeder J, Kearns G, Bertino J. Cyp2c9 mutant alleles frequencies in a rural us Caucasian population. Clin Pharmacol Ther. 2000;67(2):120.

34. Choo E, Dresser G, Stein C, et al. Identification of a new CYP2C9 variant in African-Americans. Clin Pharmacol Ther. 2000;67(2):169.

35. Jittikoon J, Mahasirimongkol S, Charoenyingwattana A, et al. Comparison of genetic variation in drug ADME-related genes in Thais with Caucasian, African and Asian HapMap populations. J Hum Genet. 2016;61(2):119-127. doi:10.1038/jhg.2015.115

36. Villegas-Torres B, Sánchez-Girón F, Jaramillo-Villafuerte K, Soberón X, Gonzalez-Covarrubias V. Genotype frequencies of VKORC1 and $\mathrm{CYP} 2 \mathrm{C} 9$ in native and Mestizo populations from Mexico, potential impact for coumarin dosing. Gene. 2015;558 (2):235-240. doi:10.1016/j.gene.2014.12.068

37. Céspedes-Garro C, Fricke-Galindo I, Naranjo MEG, et al. Worldwide interethnic variability and geographical distribution of CYP2C9 genotypes and phenotypes. Expert Opin Drug Metab Toxicol. 2015;11 (12):1893-1905. doi:10.1517/17425255.2015.1111871
38. Aithal GP, Day CP, Kesteven PJ, Daly AK. Association of polymorphisms in the cytochrome P450 CYP2C9 with warfarin dose requirement and risk of bleeding complications. Lancet. 1999;353 (9154):717-719. doi:10.1016/S0140-6736(98)04474-2

39. Gaedigk A, Casley WL, Tyndale RF, Sellers EM, Jurima-Romet M, Leeder JS. Cytochrome P4502C9 (CYP2C9) allele frequencies in Canadian Native Indian and Inuit populations. Can $J$ Physiol Pharmacol. 2001;79(10):841-847. doi:10.1139/y01-065

40. Holstein A, Plaschke A, Ptak M, et al. Association between CYP2C9 slow metabolizer genotypes and severe hypoglycemia on medication with sulphonylurea hypoglycaemic agents. $\mathrm{Br} J$ Clin Pharmacol. 2005;60(1):103-106. doi:10.1111/j.1365-2125.2005.02379.x

41. Surendiran A, Pradhan S, Agrawal A, et al. Influence of CYP2C9 gene polymorphisms on response to glibenclamide in type 2 diabetes mellitus patients. Eur J Clin Pharmacol. 2011;67(8):797-801. doi:10.1007/s00228-011-1013-8

42. Kirchheiner J, Roots I, Goldammer M, Rosenkranz B, Brockmöller J. Effect of genetic polymorphisms in cytochrome P450 (CYP) 2 C9 and CYP2C8 on the pharmacokinetics of oral antidiabetic drugs. Clin Pharmacokinet. 2005;44(12):1209-1225. doi:10.2165/00003088200544120-00002

43. Kim YM, Yoo SH, Kang RY, et al. Identifying drugs needing pharmacogenetic monitoring in a Korean hospital. Am J Health Syst Pharm. 2007;64(2):166-175. doi:10.2146/ajhp050490

44. Mosikian A, Dolgorukova A, Zalevskaya A. Possible approaches to CYP2C9-guided prescription of sulfonylureas in Russia. Pharmacogenomics. 2016;17(18):2115-2126. doi:10.2217/pgs-20160121

45. Tacconelli S, Patrignani P. Inside epoxyeicosatrienoic acids and cardiovascular disease. Front Pharmacol. 2014;5:239. doi:10.3389/ fphar.2014.00239
Vascular Health and Risk Management

\section{Publish your work in this journal}

Vascular Health and Risk Management is an international, peerreviewed journal of therapeutics and risk management, focusing on concise rapid reporting of clinical studies on the processes involved in the maintenance of vascular health; the monitoring, prevention and treatment of vascular disease and its sequelae; and the involvement

\section{Dovepress}

of metabolic disorders, particularly diabetes. This journal is indexed on PubMed Central and MedLine. The manuscript management system is completely online and includes a very quick and fair peerreview system, which is all easy to use. Visit http://www.dovepress. com/testimonials.php to read real quotes from published authors. 